\begin{tabular}{|c|l|}
\hline IV Simpósio Paranaense de Modelagem, & Artigo: \\
$\begin{array}{c}\text { Simulaçãoe Controle de Processos } \\
\text { ISSN : 1984-7521 }\end{array}$ & 12 \\
& Páginas: $\mathbf{8 2}$ - $\mathbf{8 8}$ \\
\hline
\end{tabular}

\title{
Modeling of flow through bundle of tubes using porous media approach
}

\author{
Isadora Bussi, Jaci Carlo Schramm Câmara Bastos, Henry França Meier, Leonardo Machado da \\ Rosa*
}

Regional University of Blumenau-FURB-SC, leorosa@gmail.com

\begin{abstract}
The computational fluid dynamics technique can be applied in different areas of engineering, including the observation of industrial equipment phenomena. In boilers recovery, the pressure drop due to bundle of tubes is an important parameter that can be analyzed through numerical simulations. In this study, simulations considering bundle of tubes were run in order to calibrate a porous media that represented these tubes. The complexity and time needed to develop meshes considering porous media are considerably lower, justifying its application. Once the media calibration is done, the resulting pressure drop at both configurations showed good agreement, proving the viability of using porous media in industrial applications.

Keywords: Porous media. Computational Fluid Dynamics (CFD). Bundle of tubes.
\end{abstract}

\section{Introduction}

Boilers recovery are equipment that operate through heat transfer between fluids, usually from flue gases to water. The energy required to boil and heat the water serves as heat source for industrial processes. There are different types of boilers, being the fire tubes and water tubes the most common types. In both configurations' combustion gases flow through the bundle of tubes, heating the fluid in the process.

The Computational Fluid Dynamics (CFD) is a technique that can be used to study processes in boilers, making possible to observe inside and outside phenomena. E.g., Li et al (2013) confirmed through simulations the impact of deposits in bundle of tubes during heat transfer and steel surface temperature. CFD can predict the flow, mixtures, combustion, transfers and heat loss in boilers [6]. Therefore, CFD is suitable for studies of industrial equipment operations, highlighting boilers.

For the present study, considering the flow through bundle of tubes such as those present in boilers, more efficient ways for flow representation are sought. As shown in literature $[1,2,4]$, similar cases need complex meshes, which need more time for its development. Thus, in this study we evaluate the possibility of using a porous media, hence enabling the use of relatively simpler meshes once the porous media is properly calibrated. The porous media represents the resistance to flow imposed, in this case by the bundle of tubes.

Low values for heat transfer coefficient could be related to material deposits and/or corrosion in the tubes, as well the presence of obstacles in the flow [5]. Hence, the pressure drop is a variable that requires to be analyzed to verify the efficiency of boilers. The pressure drop is caused by flow disturbances. They can affect the velocity around the tubes, consequently affecting heat transfer, compromising the boiler operation.

With the pressure drop values, is possible to calibrate a porous media, which is the objective of this study. Once the porous media is applied, the time and effort needed to carry out numerical simulations are reduced. 


\section{Numerical Simulations}

With the purpose of evaluating the applicability of porous media approach, simulations considering different horizontal $(\Delta \mathrm{x})$ and vertical $(\Delta \mathrm{y})$ distances between the tubes with a diameter $\mathrm{D}$ were run. All possible dispositions for the bundle simulations are shown in Table 1. All simulations considered a reference velocity, as well as half and double of this value, totaling 27 simulations.

Table 1. Bundle of tubes configurations.

\begin{tabular}{|c|c|c|c|}
\hline $\begin{array}{ll} & \text { Horizontal } \\
\text { Vertical } & \end{array}$ & $0.5 \mathrm{D}$ & $1.0 \mathrm{D}$ & $1.5 \mathrm{D}$ \\
\hline $0.5 \mathrm{D}$ & Case 1 & Case 4 & Case 7 \\
\hline $1.0 \mathrm{D}$ & Case 2 & Case 5 & Case 8 \\
\hline $1.5 \mathrm{D}$ & Case 3 & Case 6 & Case 9 \\
\hline
\end{tabular}

The diameter $(\phi)$ value considered in this study was $20 \mathrm{~mm}$, and the velocities as 5,10 and $20 \mathrm{~m} / \mathrm{s}$. The bundle of tubes is composed by a total of 50 tubes, arranged in 5 columns and 10 lines. A representation of the tubes disposition can be seen in Figure 1.

Figure 1. Schematic diagram of the bundle of tubes dimensions.
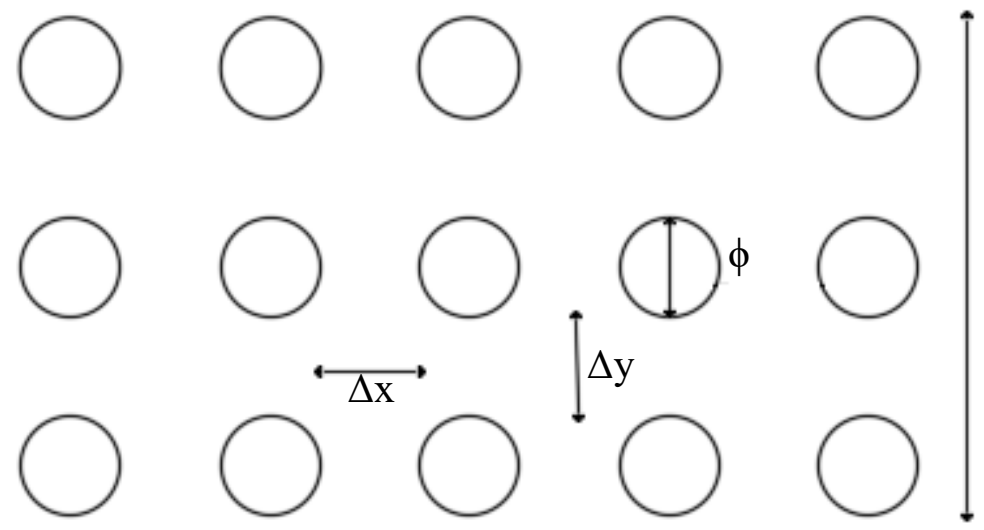

$\mathrm{L}$

It was considered that the boiler operating under $917^{\circ} \mathrm{C}$ and atmospheric pressure. In these conditions, the fluid (flue gas) has a density of $0.2928 \mathrm{~kg} / \mathrm{m}^{3}$ and viscosity of $4.71 \times 10^{-5} \mathrm{~kg} /(\mathrm{m} . \mathrm{s})[8]$.

The mathematical model is based on the mass and momentum conservation equations. The Finite Volumes Method were used for the numeric solution in microscale Navier-Stokes and continuity equations, in a two-dimensional approach. The pressure drop between was analyzed in macroscale, considering the inlet and outlet domain of the flow function [7].

The following assumptions were made:

- isothermal flow;

- gases characterized by medium properties in each region;

- each bundle of tube can be modeled defining "porous" regions, in which the flow is restricted according to Darcy-Forchheimer law.

The continuity and momentum transport equations are expressed by the Equations (1) and (2), respectively: 
$\frac{\partial \rho_{\mathrm{g}}}{\partial \mathrm{t}}+\nabla \cdot\left(\rho_{\mathrm{g}} \mathbf{v}_{\mathrm{g}}\right)=0$

$\frac{\partial}{\partial \mathrm{t}}\left(\rho_{\mathrm{g}} \mathbf{v}_{\mathbf{g}}\right)+\nabla \cdot\left(\rho_{\mathrm{g}} \mathbf{v}_{\mathbf{g}} \mathbf{v}_{\mathrm{g}}\right)=-\nabla \cdot\left(\mathbf{T}_{\mathbf{g}}^{\mathbf{e f}}\right)-\nabla \mathrm{p}+\mathbf{F}_{\text {res }}$

The density $\left(\rho_{\mathrm{g}}\right)$ and velocity $\left(v_{\mathrm{g}}\right)$ refer to the gas phase. The effective stress tensor $\left(T_{g}^{e f}\right)$ is similar to a Newtonian fluid model, being directly proportional to deformation rate and it is expressed by:

$\mathbf{T}_{\mathbf{g}}^{\mathbf{e f}}=-2 \mu_{\mathrm{g}}^{\mathrm{ef}} \mathbf{D}_{\mathbf{g}}$

The strain rate tensor is:

$\mathbf{D}_{\mathbf{g}}=\frac{1}{2}\left[\nabla \mathbf{v}_{\mathbf{g}}+\left(\boldsymbol{\nabla} \mathbf{v}_{\mathbf{g}}\right)^{\mathrm{T}}\right]$

The effective viscosity of gas phase $\left(\mu_{g}^{e f}\right)$ is composed by a laminar contribution $\left(\mu_{g}^{l}\right)$ and a turbulent one $\left(\mu_{g}^{t}\right)$,

$\mu_{\mathrm{g}}^{\mathrm{ef}}=\mu_{\mathrm{g}}^{(\mathrm{l})}+\mu_{\mathrm{g}}^{(\mathrm{t})}$

The turbulent viscosity was estimated through the SST k-omega turbulent model, which is based on the k-epsilon and k-omega models. In this model, it is assumed that the turbulent viscosity is linked to the turbulent kinect energy and turbulent vorticity (both transported properties) through the relation:

$\mu_{\mathrm{g}}^{(l)}=\rho_{\mathrm{g}} \frac{\mathrm{kg}_{\mathrm{g}}}{\omega_{\mathrm{g}}}$

In Equation 2, $F_{\text {res }}$ refers to a sum of external forces acting on the flow. In single phase simulations, this force sums up as the resistance imposed by the bundle of tubes, in the delimited regions. The modeling of this force considered the viscous and inertial contributions as expressed in Darcy-Forchheimer law:

$\mathbf{F}_{\text {res }}=\frac{-\mu_{\mathrm{g}}}{\mathrm{K}_{\text {perm }}} \mathbf{v}_{\mathbf{g}}-\mathrm{K}_{\text {loss }} \frac{\rho}{2}\left|\mathbf{v}_{\mathbf{g}}\right| \mathbf{v}_{\mathbf{g}}$

In Equation (7), $\mathrm{K}_{\text {perm }}$ refers to the media permeability, that is a proportionality constant between the pressure gradient and fluids medium velocity in viscous regime. $\mathrm{K}_{\text {loss }}$ refers to the loss due to kinect effects. Knowing the parameters for the DarcyForchheimer model, it is possible to simulate the flow through a bundle of tubes adopting a porous region, that can correctly predict the pressure drop promoted by the tubes.

The software used in this study were ICEM to elaborate the meshes and Fluent for the simulations, both in 19.0 version. The simulations were run in a CORE i3 computer, with a $2.3 \mathrm{GHz}$ processor and a 4 GB RAM memory. The calculations were performed first in steady state during 100 interactions, and then in transient state till it reaches around 2000 interactions taking around 30 minutes per simulation, which were enough to make oscillations in the time-averaged values negligible. The residues were under the $10^{-4}$ values.

The GCI (Grid Convergence Index) method was used to determine the meshes refinement for the simulations considering bundles of tubes geometry [3]. The adopted meshes in this study are composed by approx. 106 thousand hexahedral volumes. Besides, no wall function was considered in the simulations, enabling to quantify with 
greater precision the pressure drop due to the bundle of tubes. Therefore, the dimensionless $\mathrm{y}^{+}$value was set around 1 or less, for every evaluated condition.

To calculate the pressure drop in each case, the differences between inlet and outlet pressure were used. With the pressure drop and bundle length (L), it was possible to calculate the linear pressure drop $(\mathrm{Pa} / \mathrm{m})$, which was used to calibrate the porous media parameters. The porous media calibration relays on two constants, related with the fluid permissiveness and viscosity regarding the media. These values were established through the minimum squares method, which required that the DarcyForchheimer relation (Equation 7) be rewritten as a second order polynomial equation:

$$
\frac{\Delta \mathrm{P}}{\mathrm{L}}=\mathrm{av} \mathrm{v}^{2}+\mathrm{bv}
$$

\section{Results and Discussion}

The simulated results for the fluid velocity and pressure drop were first analyzed in the configuration with both horizontal and vertical distances equal to the tubes diameter (Case 5).

The flow fields through the bundle of tubes corresponding to the different inlet velocities are illustrated in Figure 2. The fluid behavior, even while suffering alterations in its velocities, remains the same: the velocity is intensified in lateral regions around the tubes. In these regions, the fluid accelerates and, consequently, the pressure is reduced.

Figure 2. Velocity fields predicted for the inlet velocities of (a) $5 \mathrm{~m} \cdot \mathrm{s}^{-1}$, (b) $10 \mathrm{~m} \cdot \mathrm{s}^{-1}$ and (c) $20 \mathrm{~m} . \mathrm{s}^{-1}$.

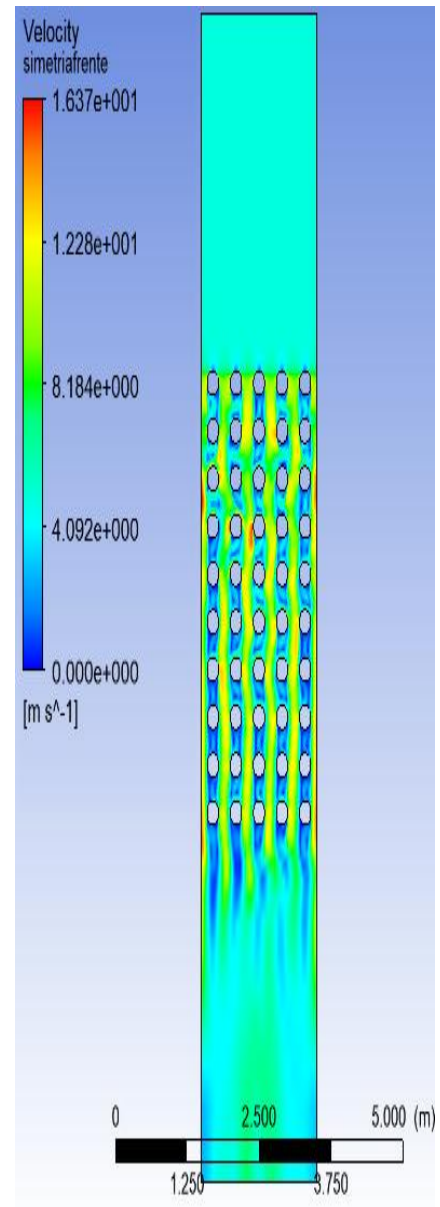

(a)

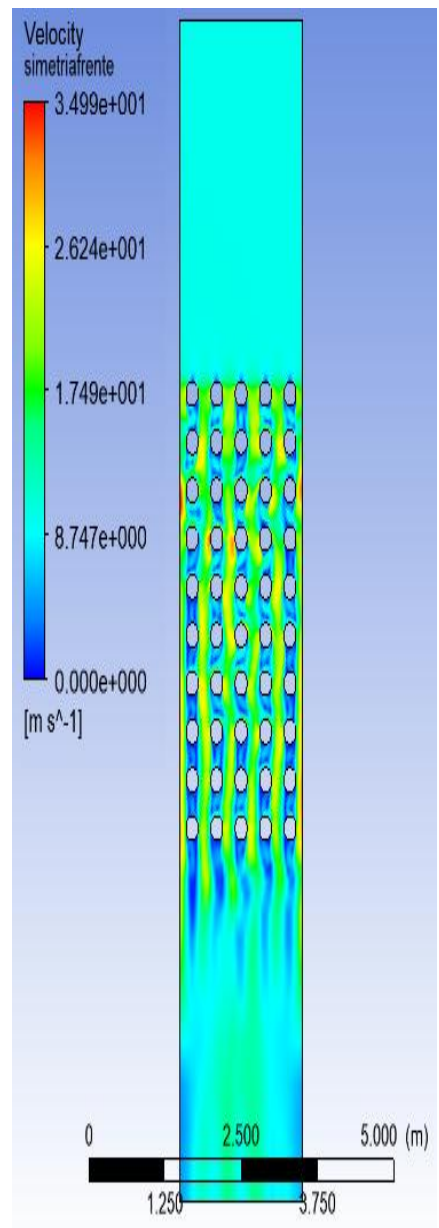

(b)

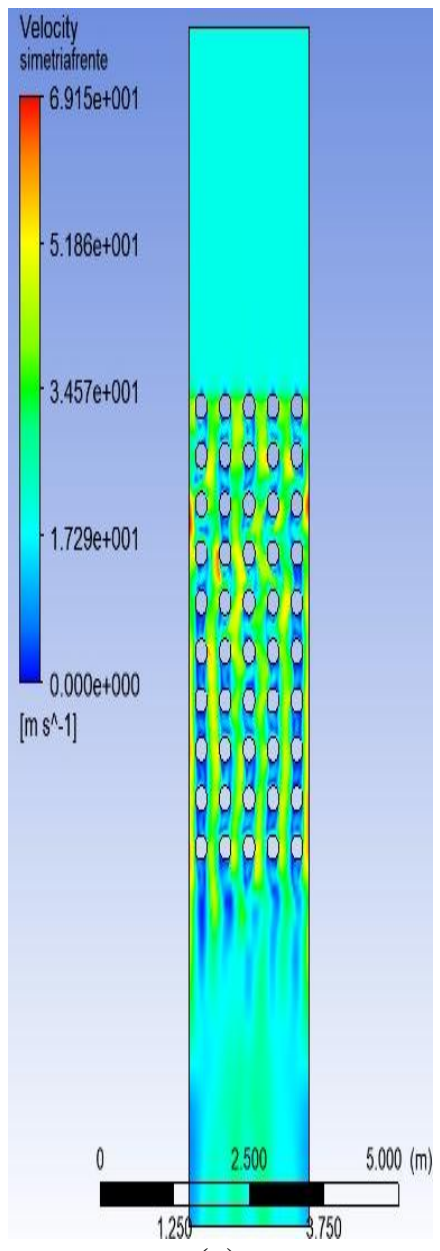

(c) 
The distances between the tubes show some direct influence among the observed variables during the flow in boilers. This statement is proved when made a comparation between Case 1, 2 and 4, it was noticed that increases in the horizontal distances (Case 1 and 4) did not affected significantly the maximum velocity, whose results were 36.8 $\mathrm{m} / \mathrm{s}$ and $36.7 \mathrm{~m} / \mathrm{s}$ for Cases 1 and 4, respectively. The same analysis was made for Cases 1 and 2 to evaluate the influence of the vertical distance. An increase on maximum velocity was observed, as the fluid reached values of $41.4 \mathrm{~m} / \mathrm{s}$. Thus, it is possible to affirm that the horizontal distances do not interfere significantly in the flow pattern, when compared to the influence of the vertical distances.

To calculate the pressure drop for this case, the difference between the inlet and outlet pressure in the bundle of tubes were used. As expected, the higher the velocity, higher the pressure drop observed. As can be seen in Figure 3, the pressure gradient is located along the bundle of tubes, and the change in the inlet velocity resulted only in a change in the pressure intensity.

Figure 3. Pressure drop for inlet velocities (a) $5 \mathrm{~m} \cdot \mathrm{s}^{-1}$, (b) $10 \mathrm{~m} \cdot \mathrm{s}^{-1}$ and (c) $20 \mathrm{~m} \cdot \mathrm{s}^{-1}$.

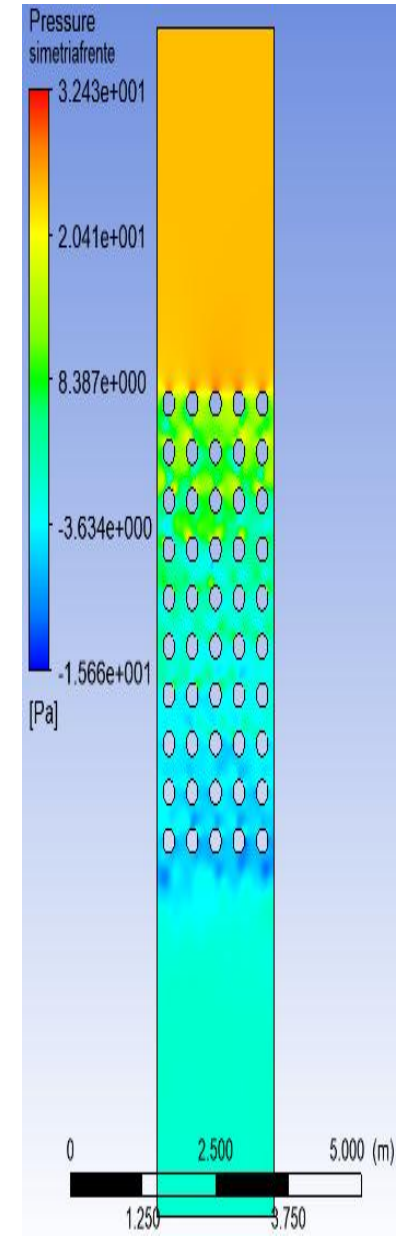

(a)

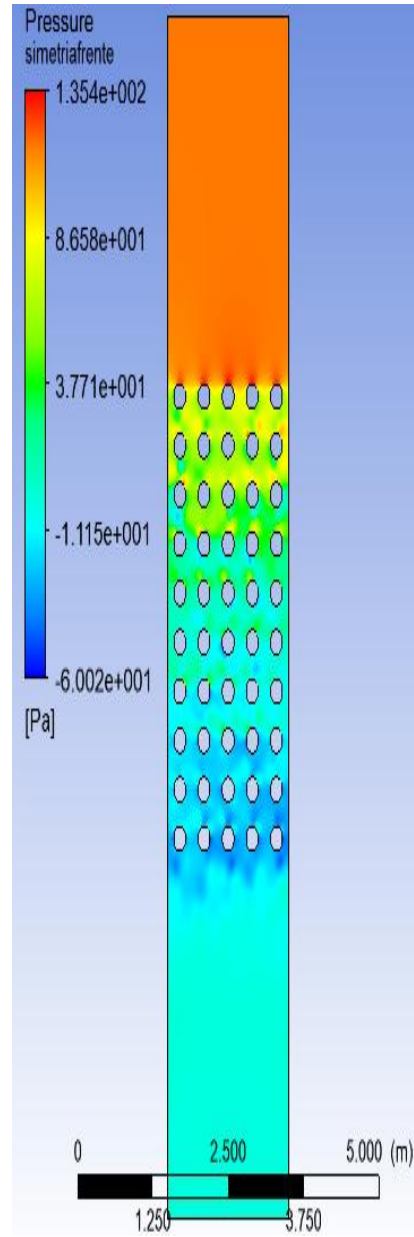

(b)

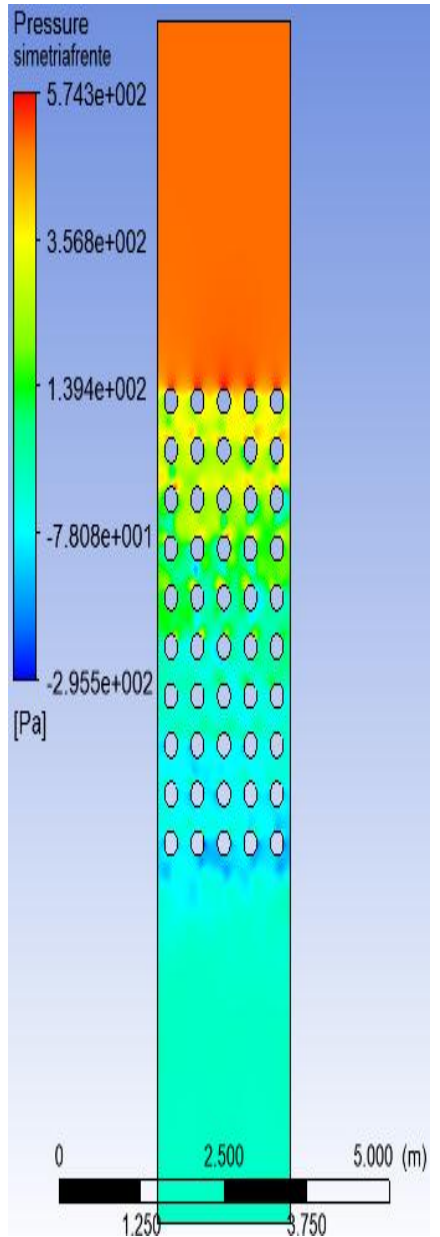

(c)

Once defined the pressure drop values and with the value for the bundle of tubes length, the linear pressure drop $(\mathrm{Pa} / \mathrm{m})$ values were calculated. The linear pressure drop was then used to obtain the parameters that determine the characterization of the porous media constants. The values of parameters a and b (Equation 8) that constitute the porous media for each configuration of bundle of tubes are presented in Table 2. 
Table 2. Extracted parameters a and $\mathrm{b}$ for each simulation.

\begin{tabular}{|l|l|l|l|l|l|l|l|l|l|}
\hline Cases & $\mathbf{1}$ & $\mathbf{2}$ & $\mathbf{3}$ & $\mathbf{4}$ & $\mathbf{5}$ & $\mathbf{6}$ & $\mathbf{7}$ & $\mathbf{8}$ & $\mathbf{9}$ \\
\hline $\mathbf{a}$ & 5.2854 & 7.8847 & 7.1621 & 2.6343 & 3.2846 & 2.4401 & 1.6832 & 1.8160 & 1.2667 \\
\hline $\mathbf{b}$ & 7.3682 & 0.7689 & 6.4084 & 0.0000 & 0.0000 & 0.0000 & 0.0000 & 0.0000 & 1.6265 \\
\hline
\end{tabular}

It can be observed a correlation between the horizontal distances of the tubes and the extracted parameter "a". As the tubes spacing increases, the parameter "a" values decreases. The same cannot be said when observing the variable $b$ and vertical distances.

With these parameters established, is possible to reproduce each simulating condition using a porous media instead of a bundle of tubes. The results for linear pressure drop obtained with simulations considering a bundle of tubes, simulations considering a porous media, and using Equation 8 are shown in Figure 4.

Figure 4. Comparation between linear pressure drop values achieved by simulations and Equation 8.

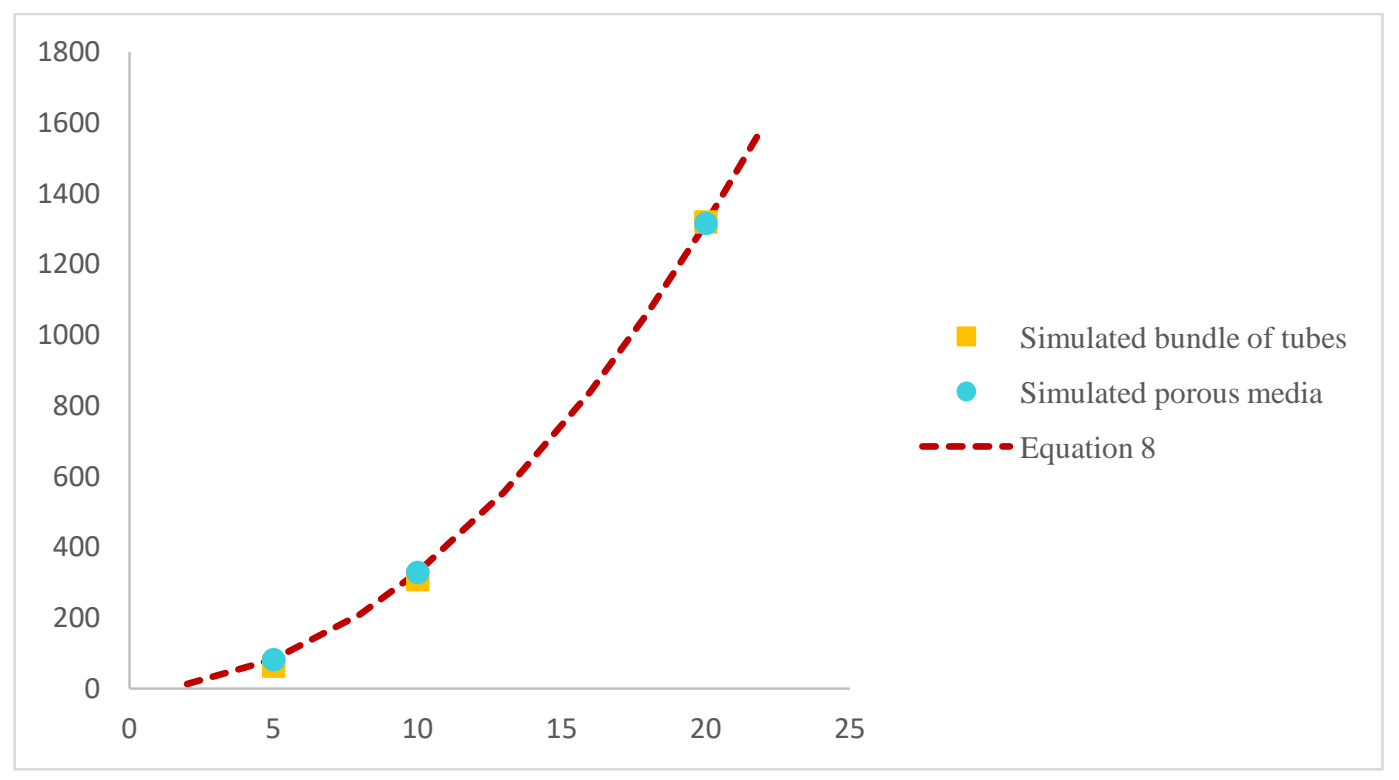

The agreement between the values obtained for linear pressure drop using different approaches must be emphasized. The greater the velocity, and, consequently, greater the pressure drop, the error of the simulations is lower. Comparing the time needed for mesh preparation, the porous media required 1/6 of the time dedicated to prepare a mesh with a bundle of tubes. Moreover, meshes considering porous media required less control volumes, which enabled the simulations to be concluded faster. However, the complexity of the porous media definition dwell in establishing properly the parameters $a$ and $b$.

\section{Conclusion}

The use of porous media is a viable alternative to simulate bundles of tubes, since the results obtained for pressure drop are close to the values obtained from the simulations with the presence of tubes. Both the time and complexity regarding the 
development of the porous media mesh were reduced once compared to the bundle of tubes simulations. Therefore, it is possible to apply this approach in studies with aligned tubes present in boilers.

\section{Acknowledgements}

Authors are grateful to the Personal Improvement in Higher Level Coordination - Brazil (CAPES) for partially funded this study, Brazilian Petroleum S.A. (Petrobras) for financial support and $\mathrm{CNPq}$ for the research scholarship fixed by $\mathrm{RN}-017 / 2006$.

\section{References}

1. Alvarado, D., Marsden Jr, S. S. (1979). Flow of oil-in-water emulsions through tubes and porous media. Society of Petroleum Engineers Journal, 19(06), 369-377.

2. Bear, J. (2013). Dynamics of fluids in porous media. Courier Corporation.

3. Celik, I. B., Ghia, U., Roache, P. J., Christopher. Procedure for estimation and reporting of uncertainty due to discretization in CFD applications Journal of fluids Engineering-Transactions of the ASME, Vol. 130, No. 7. (July 2008), doi:10.1115/1.2960953.

4. Dahle, H. K., Celia, M. A., Hassanizadeh, S. M. (2005). Bundle-of-tubes model for calculating dynamic effects in the capillary-pressure-saturation relationship. Transport in Porous media, 58(1-2), 5-22.

5. Li, B., Brink, A., Hupa, M. (2013). CFD investigation of slagging on a super-heater tube in a kraft recovery boiler. Fuel processing technology, 105, 149-153.

6. Ortiz, F. G. (2011). Modeling of fire-tube boilers. Applied Thermal Engineering, 31(16), 3463-3478.

7.Tosco, T., Marchisio, D.L., Lince, F. et al. Transp Porous Med (2013) 96: 1. https://doi.org/10.1007/s11242-012-0070-5

8.Zhang, N., Lu, B., Wang, W., Li, J., 2010. 3D CFD simulation of hydrodynamics of a 150MWe circulating fluidized bed boiler. Chem, Eng. J. 162, 821-828. 